\title{
Characterization of the role of the RadS/RadR two-component system in the radiation resistance of Deinococcus radiodurans
}

\author{
Correspondence \\ Hari S. Misra \\ hsmisra@barc.gov.in
}

Received 28 February 2011

Revised 29 June 2011

Accepted 6 July 2011

\author{
Shruti S. Desai, ${ }^{1,2}$ Yogendra S. Rajpurohit, ${ }^{1}$ Hari S. Misra ${ }^{1}$ \\ and Dileep N. Deobagkar ${ }^{2}$ \\ ${ }^{1}$ Molecular Biology Division, Bhabha Atomic Research Centre, Mumbai 400085, India \\ ${ }^{2}$ Centre for Advanced Studies in Zoology, Department of Zoology, University of Pune, \\ Pune 411007, India
}

\begin{abstract}
Deinococcus radiodurans shows extraordinary tolerance to DNA damage, and exhibits differential gene expression and protein recycling. A putative response regulator, the DRB0091 (RadR) ORF, was identified from a pool of DNA-binding proteins induced in response to gamma radiation in this bacterium. radR is located upstream of $d r B 0090$, which encodes a putative sensor histidine kinase (RadS) on the megaplasmid. Deletion of these genes both individually and together resulted in hypersensitivity to DNA-damaging agents and a delayed or altered double-strand break repair. A $\Delta r a d R r a d S$ double mutant and a $\Delta r a d R$ single mutant showed nearly identical responses to gamma radiation and UVC. Wild-type RadR and RadS complemented the corresponding mutant strains, but also exhibited significant cross-complementation, albeit at lower doses of gamma radiation. The radS transcript was not detected in the $\Delta$ radR mutant, suggesting the existence of a radRS operon. Recombinant RadS was autophosphorylated and could catalyse the transfer of $\gamma$ phosphate from ATP to RadR in vitro. These results indicated the functional interaction of RadS and RadR, and suggested a role for the RadS/RadR two-component system in the radiation resistance of this bacterium.
\end{abstract}

\section{INTRODUCTION}

Deinococcus radiodurans is characterized by its extreme tolerance to DNA-damaging agents, including radiation and desiccation (Makarova et al., 2001; Blasius et al., 2008). Efficient biphasic DNA double-strand break (DSB) repair (Daly et al., 1994; Daly \& Minton, 1996) and a strong oxidative stress tolerance (Markillie et al., 1999; Slade \& Radman, 2011) are among the mechanisms attributed to these phenotypes. DSB repair is primarily supported by extended synthesis-dependent strand annealing (ESDSA) (Zahradka et al., 2006) and the RecF pathway of homologous recombination (Misra et al., 2006; Slade et al., 2009). D. radiodurans cells exposed to gamma radiation show a relatively longer lag phase. During this period cell division stops, and resumes presumably after DSB repair has been accomplished (Cox \& Battista, 2005). This might indicate the existence of an uncharacterized checkpoint regulation between DNA repair and cell division. A bacterium exposed to gamma radiation adjusts its

Abbreviations: DSB, double-strand break; PIR, post-irradiation; TCS, two-component system.

Three supplementary figures and a supplementary table are available with the online version of this paper. transcriptome and proteome by regulating gene expression (Liu et al., 2003; Tanaka et al., 2004) and protein recycling (Joshi et al., 2004). The SOS response, a classical example of a DNA-damage response in bacteria (Shimoni et al., 2009; Walker, 1996), is missing in D. radiodurans (Bonacossa de Almeida et al., 2002; Narumi et al., 2001). Recently, the involvement of a eukaryotic-type Ser/Thr protein kinase (eSTPK) in gamma radiation resistance and DSB repair of $D$. radiodurans has been demonstrated (Rajpurohit \& Misra, 2010). The roles of Ser/Thr protein kinases in DNA damage-induced signalling processes are better understood in eukaryotes (Zhou \& Elledge, 2000). Further, the genome of this bacterium encodes a large number of putative transcription factors, response regulators and different types of protein kinases, including 20 putative histidine kinases (Leonard et al., 1998; White et al., 1999; Makarova et al., 2001; Kim \& Forst, 2001). Thus, the contribution of these unique proteins to the extraordinary radiation resistance of this bacterium can be envisaged.

Here, we report the functional characterization of the DR_B0090 and DR_B0091 ORFs, encoding a putative sensor histidine kinase (hereafter referred to as RadS) and a cognate response regulator (hereafter referred to as RadR), 
respectively, and their involvement in the radiation resistance and DSB repair of D. radiodurans. $\Delta$ radS and $\triangle \mathrm{radR}$ mutants showed a higher sensitivity to DNA damage and oxidative stress produced by UV $(254 \mathrm{~nm})$ and gamma radiation, hydrogen peroxide and desiccation as compared with the wild-type. The $\Delta$ radRradS double mutant and the $\Delta r a d R$ single mutant showed nearly identical responses to both gamma and UVC radiation. The expression of wild-type RadS and RadR on a plasmid showed almost complete functional complementation in the respective mutants. Purified RadS showed autokinase activity and could phosphorylate RadR in vitro. These results suggest that the $\mathrm{rad} R$ and $\mathrm{rad} S$ genes are organized in a bicistronic operon and that the RadS/RadR twocomponent system (TCS) plays an important role in the radiation resistance of $D$. radiodurans.

\section{METHODS}

Bacterial strains, plasmids and growth conditions. D. radiodurans R1, a generous gift from Professor J. R. Battista, Louisiana State University, Baton Rouge, LA, USA, was grown in TGY medium ( $0.5 \%$ Bacto tryptone, $0.1 \%$ glucose, $0.3 \%$ Bacto yeast extract), as described earlier (Mattimore \& Battista, 1996). Plasmids pET28a + (Novagen) and pRADgro (Misra et al., 2006) and their derivatives were maintained in Escherichia coli strain DH5 $\alpha$ in Luria-Bertani (LB) broth or on LB agar plates supplemented with kanamycin $(25 \mu \mathrm{g}$ $\mathrm{ml}^{-1}$ ) and ampicillin $\left(100 \mu \mathrm{g} \mathrm{ml} \mathrm{m}^{-1}\right)$, respectively. All molecular biology grade chemicals were purchased from Sigma Chemical Co., Roche Biochemicals, New England Biolabs and Bangalore Genei. Deinococcus mutants were grown in the presence of kanamycin $(8 \mu \mathrm{g}$ $\left.\mathrm{ml}^{-1}\right)$ and chloramphenicol $\left(5 \mu \mathrm{g} \mathrm{ml} \mathrm{m}^{-1}\right)$, as required. Other recombinant techniques used were as described by Sambrook \& Russell (2001).

Isolation of DNA-binding proteins using a deinococcal DNAcellulose matrix. Genomic DNA was isolated from both unirradiated and gamma-irradiated $D$. radiodurans $\mathrm{R} 1$ as described earlier (Battista et al., 2001). DNA was sonicated at $30 \mathrm{~Hz}$, one stroke of $2 \mathrm{~s}$ with $0.2 \mu \mathrm{m}$ duty cycles, and was UV cross-linked with cellulose (DNA-cellulose). Free DNA was washed with buffer containing $50 \mathrm{mM} \mathrm{NaCl}$. The cell-free extracts were prepared from unirradiated cells and from cells after $0,15,30,45$ and $60 \mathrm{~min}$ irradiation, and were incubated with the DNA-cellulose matrix for $60 \mathrm{~min}$. The proteins bound to DNA-cellulose were packed into a column and washed with buffer A $(20 \mathrm{mM}$ Tris/HCl, pH 7.5, $20 \%$, v/v, glycerol, $0.5 \mathrm{mM}$ EDTA, pH $8,0.5 \mathrm{mM}$ PMSF, $0.5 \mathrm{mM}$ DTT) containing $50 \mathrm{mM} \mathrm{NaCl}$, to remove the unbound proteins until the $A_{280}$ was nearly zero. The bound proteins were eluted with a buffer containing $0.7 \mathrm{M} \mathrm{NaCl}$ and dialysed in buffer A supplemented with $50 \%, \mathrm{v} / \mathrm{v}$, glycerol and stored at $-20{ }^{\circ} \mathrm{C}$ until further use.

Generation of deletion mutants of $\boldsymbol{D}$. radiodurans. The $\Delta$ radS and $\Delta \mathrm{radR}$ mutants were generated using strategies described previously (Khairnar et al., 2008). The primers used in this study are listed in Supplementary Table S1. In brief, the $1 \mathrm{~kb}$ upstream of the initiation codon and $1 \mathrm{~kb}$ downstream from the stop codon of DR_B0090 (radS) were PCR-amplified using primers HK90F1 and HK90R1 for upstream, and primers HK90F2 and HK90R2 for downstream fragments. Similarly, $1 \mathrm{~kb}$ upstream and $1 \mathrm{~kb}$ downstream from DR_B0091 ( radR) were PCR-amplified using RR91F1 and RR91R1 primers for the upstream and RR91F2 and RR91R2 for the downstream fragments, separately. The upstream fragments of both genes were cloned at ApaI and EcoRI sites into pNOKOUT, and downstream fragments were cloned at $\mathrm{BamHI}$ and $\mathrm{XbaI}$ sites to yield pNOKHK90 and pNOKRR91, respectively. Similarly, the upstream fragment of radR and the downstream fragment of radS were cloned into pNOKOUT to yield pNOKradRS. These recombinant plasmids were linearized with $X m n \mathrm{I}$ and transformed into $D$. radiodurans, and transformants were grown for several generations in TGY broth containing kanamycin $\left(8 \mu \mathrm{g} \mathrm{ml}^{-1}\right)$. The complete replacement of wild-type alleles(s) with the neomycin phosphotransferase gene (nptII) was confirmed by PCR amplification using gene-specific primers, and the single mutants were designated $\Delta \mathrm{radS}$ and $\Delta \mathrm{radR}$ (Supplementary Fig. S1) and the double mutant $\Delta$ radRradS.

Construction of RadS and RadR expression plasmids. The $1.3 \mathrm{~kb}$ radS and $0.66 \mathrm{~kb}$ radR genes were PCR-amplified from the genomic DNA of $D$. radiodurans using gene-specific primers and cloned into pRADgro at the ApaI and HindIII sites (Misra et al., 2006) to obtain pGroHK90 and pGroRR91, respectively. The recombinant plasmids were transformed into $D$. radiodurans and the respective deletion mutants as described earlier (Meima et al., 2001). The transformants were scored on TGY agar plates containing chloramphenicol $\left(8 \mu \mathrm{g} \mathrm{ml}^{-1}\right)$. The $\mathrm{radS}$ and $\mathrm{radR}$ genes were PCRamplified using sequence-specific primers and cloned at the NdeI and HindIII sites into pET28a + to yield pETHK90 and pETRR91, respectively. These plasmids were transformed into E. coli BL21 (DE3) pLysS for expression and purification of recombinant proteins.

Cell survival studies. $D$. radiodurans and its derivatives were grown in TGY medium at $32{ }^{\circ} \mathrm{C}$ to the late-exponential phase and treated with different doses of gamma radiation at a dose rate of about $5.86 \mathrm{kGy} \mathrm{h}{ }^{-1}$ (G5000, ${ }^{60} \mathrm{Co}$, Board of Radiation and Isotopes Technology, Department of Atomic Energy, India), and UV $\left(254 \mathrm{~nm}\right.$ ) radiation at $0.295 \mathrm{~J} \mathrm{~s}^{-1} \mathrm{~m}^{-2}$ (Gentec PSV-3303 laser power meter), as described previously (Kota \& Misra, 2006). For desiccation, the cells were incubated at $5 \%$ humidity for different time intervals as described earlier (Mattimore \& Battista, 1996). For hydrogen peroxide, the cells were treated with different concentrations of $\mathrm{H}_{2} \mathrm{O}_{2}$ for $30 \mathrm{~min}$ with shaking. Appropriate dilutions were plated on TGY agar plates and the c.f.u. $\mathrm{ml}^{-1}$ was recorded after $48 \mathrm{~h}$ of incubation at $32{ }^{\circ} \mathrm{C}$.

DNA strand break repair studies. The cells were irradiated with $6.5 \mathrm{kGy}$ of gamma radiation at a dose rate of $5.86 \mathrm{kGy} \mathrm{h}^{-1}$ and aliquots were collected at different time intervals post-irradiation (PIR). The DNA fragments were separated by PFGE using the modified protocols described by Mattimore \& Battista (1996). In brief, the cells were washed with PBS-saturated butanol for $30 \mathrm{~min}$ at $25{ }^{\circ} \mathrm{C}$, followed by a wash in $50 \%$ ethanol for $5 \mathrm{~min}$. Cells were embedded in $0.7 \%$ agarose and plugs were incubated in lysis buffer I ( $5 \mathrm{mg}$ lysozyme $\mathrm{ml}^{-1}, 5 \mathrm{mM}$ EDTA, $\mathrm{pH} 8$ ) for $5 \mathrm{~h}$ at $37^{\circ} \mathrm{C}$, followed by overnight incubation at $50{ }^{\circ} \mathrm{C}$ in lysis buffer II (0.5 M EDTA, $\mathrm{pH} 8,1 \%$ sodium sarcosine, $2 \mathrm{mg}$ proteinase $\mathrm{K} \mathrm{ml}^{-1}$ ). The plugs were washed four times with TE buffer $(10 \mathrm{mM}$ Tris/HCl, $\mathrm{pH} 8.0$, 1 mM EDTA, pH 8.0) and then washed three times for $1 \mathrm{~h}$ each with NotI buffer at $50{ }^{\circ} \mathrm{C}$. Subsequently, the plugs were transferred to fresh tubes containing $30 \mathrm{U}$ Not $\mathrm{I}$ in its buffer and incubated overnight at $37^{\circ} \mathrm{C}$. The DNA fragments were analysed on $1 \%$ multipurpose agarose (Roche Biochemicals) and visualized by staining with ethidium bromide.

Purification of recombinant protein and in vitro protein phosphorylation. E. coli BL21(DE3) pLysS cells harbouring pETHK90 and pETRR91 plasmids were induced with $200 \mu \mathrm{M}$ IPTG and proteins were analysed by $10 \%$ SDS-PAGE. Recombinant proteins were purified by immobilized metal-chelating affinity 
chromatography (IMAC), using modified protocols described previously (Misra et al., 1998). In brief, the IPTG-induced cells were suspended in sample buffer $\left(100 \mathrm{mM} \mathrm{NaH} \mathrm{PO}_{4}, 10 \mathrm{mM}\right.$ Tris/HCl, $8 \mathrm{M}$ urea, $\mathrm{pH} 8.0$ ) and incubated at $37{ }^{\circ} \mathrm{C}$ for $30 \mathrm{~min}$ with mild agitation. The clear supernatant was passed through an IMAC column pre-equilibrated with sample buffer and washed with sample buffer adjusted to $\mathrm{pH}$ 6.3. Proteins were eluted with sample buffer at $\mathrm{pH} 5.9$ and analysed by $10 \%$ SDS-PAGE. Pure fractions were pooled, adjusted to $\mathrm{pH} 8.0$ and passed through a fresh IMAC column. Protein refolding was done with a slow gradient of 8-0 $\mathrm{M}$ urea containing 0 $5 \mathrm{mM}$ DTT in sample buffer, and the proteins were eluted with elution buffer (10 mM Tris/HCl, $\mathrm{pH}$ 8.0, $300 \mathrm{mM} \mathrm{NaCl}, 1 \mathrm{mM}$ DTT, $10 \mathrm{mM} \mathrm{MgCl} 2,300 \mathrm{mM}$ imidazole). Purity was checked by SDSPAGE, and pure fractions were dialysed in buffer containing $10 \mathrm{mM}$ Tris/HCl, pH 8.0, $300 \mathrm{mM} \mathrm{NaCl}, 1 \mathrm{mM}$ DTT, $10 \mathrm{mM} \mathrm{MgCl}_{2}$ and $50 \%(\mathrm{v} / \mathrm{v})$ glycerol.

The kinase activity was checked by incubating the recombinant RadS and RadR separately and together in the presence of $\left[\gamma^{33} \mathrm{P}\right] \mathrm{ATP}$, as described elsewhere (Shrivastava et al., 2007). In brief, both the proteins were incubated in a reaction mixture containing $50 \mathrm{mM}$ Tris/HCl, pH 8.0, $20 \mathrm{mM} \mathrm{MgCl}_{2}, 2 \mathrm{mM}$ cold ATP and $200 \mu \mathrm{Ci}$ (7.4 MBq) $\left[\gamma^{33} \mathrm{P}\right] \mathrm{ATP}$ for different time intervals at $25{ }^{\circ} \mathrm{C}$. RadS and RadR were taken separately as controls. A dose-dependent assay was carried out with a constant RadS concentration $(20 \mu \mathrm{M})$ and an increasing concentration of RadR under similar conditions. Both RadS alone and RadS with RadR were incubated for different time periods in the presence of $\left[\gamma^{32} \mathrm{P}\right] \mathrm{ATP}$. The reaction products were analysed by $8 \%$ SDS-PAGE at $25^{\circ} \mathrm{C}$. The gel was stained with Coomassie brilliant blue and dried, and signals were recorded by autoradiography and by scanning on a PhosphorImager (Molecular Dynamics).

\section{RESULTS AND DISCUSSION}

\section{A putative response regulator can be identified amongst the radiation-induced proteins}

Cell-free extracts were prepared from unirradiated as well as gamma radiation-treated cells collected at $15 \mathrm{~min}$ intervals up to $1 \mathrm{~h}$ PIR, and DNA-binding proteins were isolated using DNA-cellulose columns, as described in Methods. Both SDS-PAGE and 2D PAGE analysis showed the differential expression of several proteins, which were identified by MALDI MS (data not shown). A protein of $\sim 28 \mathrm{kDa}$ that was induced in the first $15 \mathrm{~min}$ and maintained at higher levels up to $1 \mathrm{~h}$ PIR (Fig. 1) showed a peptide mass fingerprint (Supplementary Fig. S2) that matched with the protein encoded by the DR_B0091 ORF. A domain search analysis using www.expasy.ch/prosite indicated that this protein had a CheY-homologous receiver domain at the $\mathrm{N}$ terminus and a transcriptional regulatory protein domain at the $\mathrm{C}$ terminus (Supplementary Fig. S3). This suggested that the DRB0091 protein could interact with both protein and DNA simultaneously. The CheY receiver domain at the $\mathrm{N}$ terminus of such proteins undergoes phosphorylation at a specific aspartate residue and plays an important role in the signal transduction mechanism (Appleby et al., 1996). DR_B0090 ( radS) is located downstream from DR_B0091 $(\mathrm{rad} R)$ on the megaplasmid, and encodes a putative histidine kinase (White et al., 1999). It was predicted that

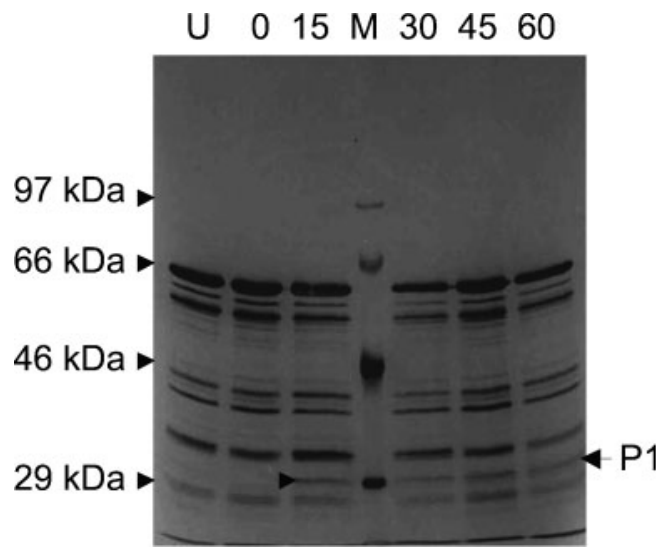

Fig. 1. SDS-PAGE analysis of dsDNA-binding proteins (DBPs) during the early phase of PIR recovery of $D$. radiodurans $\mathrm{R} 1$. Total DBPs were isolated from gamma-irradiated cells as described in Methods. DBPs from an unirradiated sample (U) and after different periods PIR $(0,15,30,45$ and $60 \mathrm{~min})$ were separated by SDSPAGE. A protein $(P 1)$ showing inducible synthesis from $15 \mathrm{~min}$ PIR onwards was identified using MALDI-TOF MS data, as shown in Supplementary Fig. S2.

the aspartyl group present at position 51 of RadR, similar to the CheY receiver domain, was the probable site for phosphorylation by RadS or other histidine kinases in this bacterium. Functional domain analysis of RadS showed the presence of HATPase and HisKA domains, and a transmembrane domain (between amino acids 7 and 29) that overlapped with the signal peptide of the protein (http:// smart.embl-heidelberg.de). RadS also contains a HAMP linker domain normally reported from bacterial sensor proteins such as histidine kinases, adenylyl cyclases, methylaccepting proteins and phosphatases, and chemotaxis proteins (http://pfam.sanger.ac.uk/family/PF00672.18). Protein kinases containing HAMP linker domains are integral membrane proteins and are involved in two-component signal transduction processes (Aravind \& Koonin, 1999) in bacteria. The HATPase domain contains the ATP-binding motif and a characteristic G-X-G motif, while the HisKA domain has a site for phosphorylation and a dimer interface motif. These characteristics suggest that RadS and RadR are a histidine sensor kinase and its cognate response regulator, respectively. The possibility that RadR is phosphorylated by RadS and that the functional interaction of these proteins contributes to the radiation resistance of this bacterium were further investigated.

\section{The $\Delta \mathrm{rad} S$ and $\Delta \mathrm{rad} R$ mutants are hypersensitive to both oxidative stress and DNA damage}

The radS and $\mathrm{radR}$ genes were deleted individually and together from the genome of $D$. radiodurans, and mutants were designated $\Delta \mathrm{radS}, \Delta \mathrm{radR}$ and $\Delta \mathrm{radSradR}$, respectively. The cell survival of single mutants was determined in response to various DNA-damaging agents such as gamma 
radiation, UVC $(254 \mathrm{~nm}), \mathrm{H}_{2} \mathrm{O}_{2}$ and desiccation. The tolerance of these cells decreased by nearly two orders of magnitude in response to gamma radiation (Fig. 2a), three orders of magnitude to UVC (Fig. 2b), 10-fold to $25 \mathrm{mM}$ hydrogen peroxide (Fig. 2c) and nearly three orders of magnitude to desiccation at $5 \%$ humidity (Fig. 2d) as compared with wild-type cells. The cell survival of the radS mutant decreased more rapidly when cells were treated with a dose of up to $2 \mathrm{kGy}$ of gamma radiation as compared with doses above $2 \mathrm{kGy}$. This indicates the possibility that RadS controls significant pathways associated with radiation resistance and DSB repair. The role of another response regulator, DR_2418, in the gamma radiation resistance of $D$. radiodurans has also been shown recently (Wang et al., 2008). These results suggest the involvement of the RadS/RadR system in the DNA damage tolerance of $D$. radiodurans.

\section{Wild-type proteins show functional complementation in trans}

To confirm that the phenotypes of the $\Delta \mathrm{rad} S$ and $\Delta \mathrm{radR}$ mutants were due to the absence of the respective proteins, the wild-type RadS and RadR were expressed on plasmids pGroHK90 and pGroRR91, respectively, in both the single and the double mutants of $D$. radiodurans. The effects of these proteins on the gamma radiation response of the mutants were evaluated. The $\Delta \mathrm{rad} S$ and $\Delta \mathrm{radR}$ mutants expressing the respective proteins recovered nearly 80 $90 \%$ of wild-type tolerance to gamma radiation (Fig. 3a, b). Interestingly, the $\Delta \mathrm{radS}$ mutant expressing RadR and the $\Delta r a d R$ mutant expressing RadS on plasmids also showed a dose-dependent recovery of DNA damage tolerance. At lower doses up to $4 \mathrm{kGy}$, the recovery of gamma radiation resistance was significant, and RadR was (a)

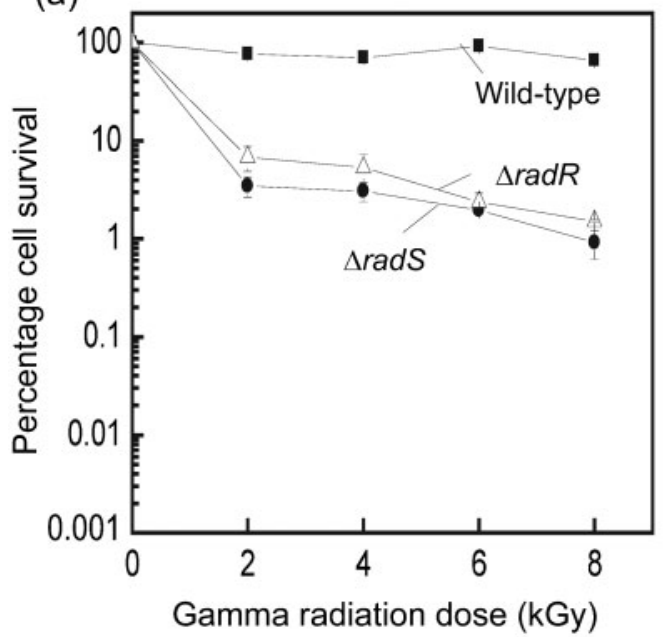

(c)

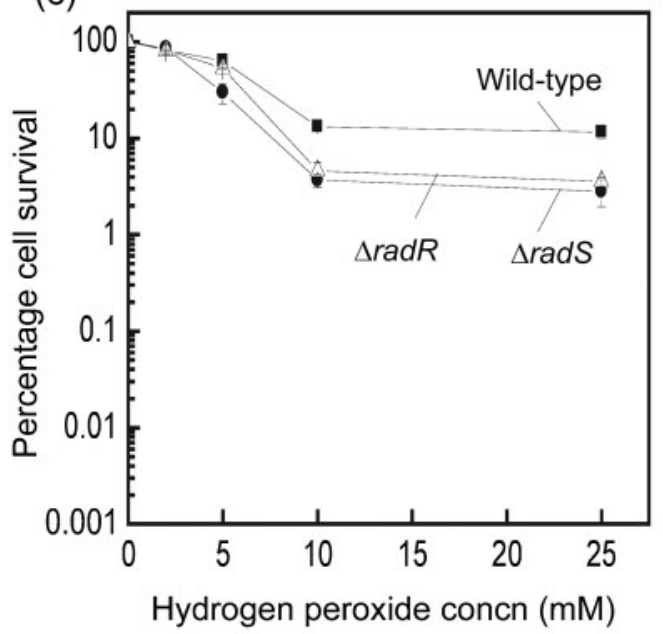

(b)

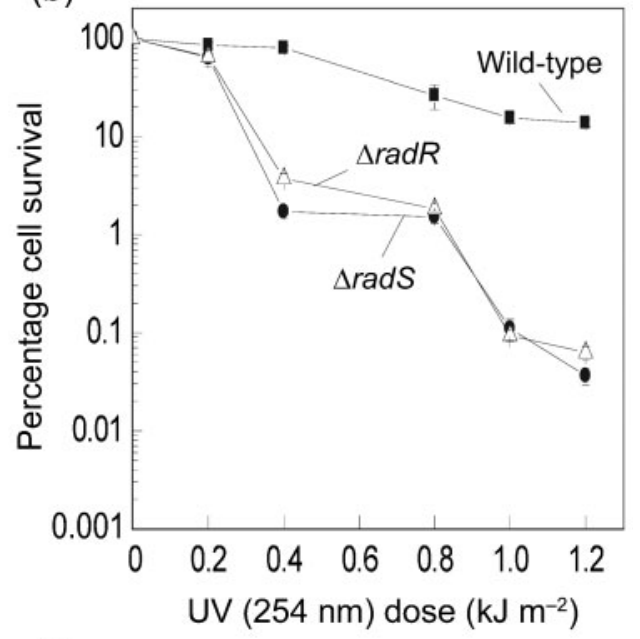

(d)

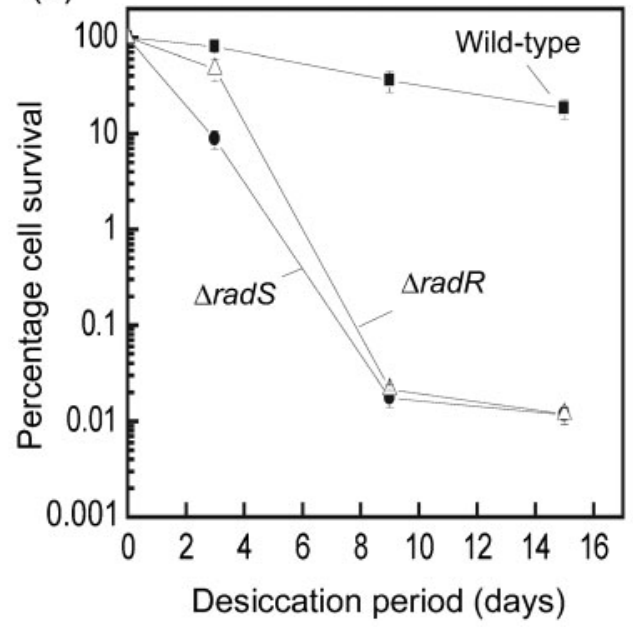

Fig. 2. DNA damage response of wild-type and mutant derivatives of $D$. radiodurans. The cell survival of wild-type, $\Delta d r B 0090$ $(\Delta \mathrm{radS})$ and $\Delta d r B 0091$ ( $\Delta$ radR) mutants was measured with gamma radiation (a), UV (b), hydrogen peroxide (c) and desiccation (d) treatments. A cell survival of $100 \%$ for the wild-type, $\Delta \mathrm{rad} R$ and $\Delta$ radS mutants corresponds to $2.8 \times 10^{7}$,

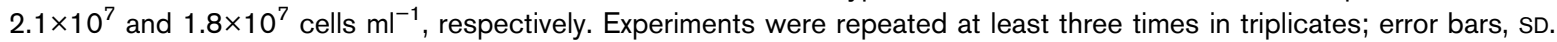



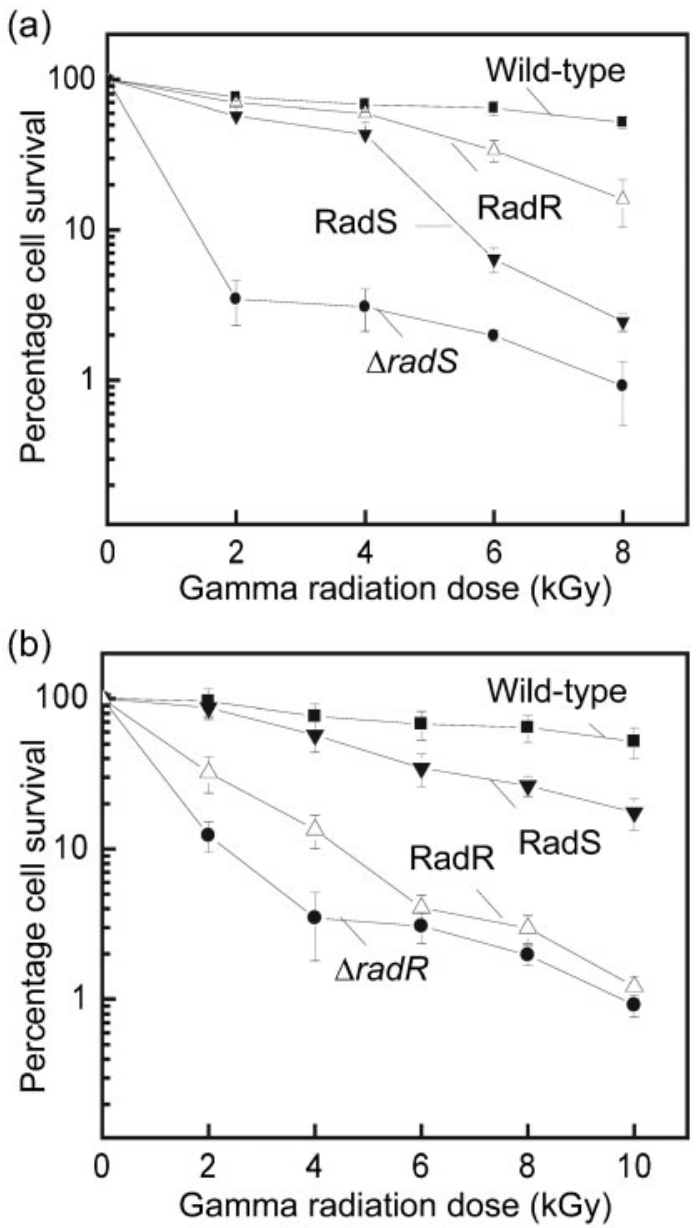

Fig. 3. Functional complementation in mutants. $\Delta r a d S$ (a) and $\Delta \operatorname{rad} R$ (b) mutants expressing wild-type radS (RadS) and radR (RadR) genes were treated with different doses of gamma radiation, and cell survival was compared with that of the wildtype. A cell survival of $100 \%$ corresponds to $3.1 \times 10^{7}, 1.5 \times 10^{7}$ and $2.7 \times 10^{7}$ cells $\mathrm{ml}^{-1}$ for the wild-type, mutants and complemented cells, respectively. Experiments were repeated at least three times in triplicates; error bars, SD.

able fully to restore the wild-type resistance to gamma radiation in the radS mutant. However, above $4 \mathrm{kGy}$, the levels of complementation in the respective mutants were significantly lower (Fig. 3). Furthermore, the deletion of the radR-radS (drB0091-drB0090) operon showed a response to UVC and gamma radiation nearly identical to that of the radR single mutant (Fig. 4). Interestingly, the expression of the wild-type alleles of radS and $\mathrm{radR}$ separately in a $\Delta$ radRradS double mutant also showed functional complementation very similar to the effects of these proteins in the $\Delta \mathrm{radR}$ mutant (Fig. 4a). However, the $\Delta$ radRradS mutant expressing the RadR protein showed a near-complete recovery of UVC resistance at lower doses, although the recovery was significantly lower at higher doses (Fig. 4b). Unlike RadS complementation with respect
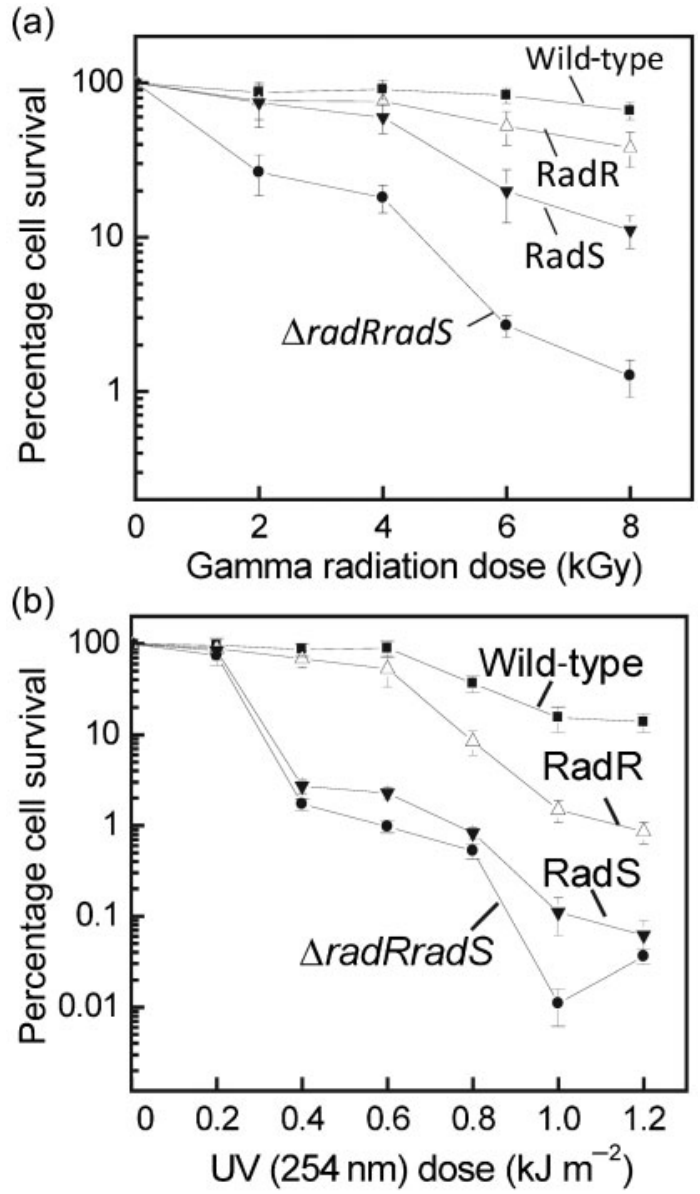

Fig. 4. Effect of DNA damage on cell survival of the $\Delta$ radRradS double mutant. The $\Delta$ radRradS mutant and its derivatives expressing wild-type $\mathrm{rad} S$ (RadS) and $\mathrm{rad} R$ (RadR) alleles were treated with different doses of gamma radiation (a) and UV (254 nm) (b), and cell survival was monitored as described in Methods and compared with that of the wild-type. A cell survival of $100 \%$ for the wild-type, double mutant and its derivatives expressing RadR and RadS corresponds to $8.2 \times 10^{7}, 1.2 \times 10^{7}$, $3.6 \times 10^{7}$ and $2.6 \times 10^{7}$ cells $\mathrm{ml}^{-1}$, respectively. Experiments were repeated at least three times in triplicates; error bars, SD.

to gamma radiation resistance, the double mutant showed a low level of RadS complementation with respect to UVC resistance (Fig. 4b). This indicated that the effects of RadS on gamma radiation- and UVC-induced DNA damage repair are different.

Genetic modifications that lead to differential responses of $D$. radiodurans to gamma and UVC radiation have been reported previously (Misra et al., 2006; Khairnar et al., 2008). The expression of transgenes cloned in pRADgro under the groESL promoter has been shown in both E. coli (Kota \& Misra, 2006) and D. radiodurans (Misra et al., 2006; Khairnar et al., 2008). The functional complementation of the radS mutation by overexpression of RadR might lead one to speculate either that RadR is activated by other 
histidine kinase(s) and/or that there is a dose-dependent effect of RadR, which might have residual activity in its unphosphorylated form. However, the complementation by wild-type RadS, though partial, in the $\Delta r a d R$ and $\Delta$ radRradS mutants is intriguing, and the molecular mechanism underlying the support by RadS of the loss of RadR is not clear. However, the possibility exists that RadS phophorylates other regulatory protein(s), which might complement the functional loss of RadR/RadS in these mutants. Since the genome of $D$. radiodurans encodes a large number of stress-responsive regulatory proteins, the possibility that some of these proteins are phosphorylated by RadS and functionally complement the functions of RadR cannot be ruled out. Transcriptome studies of the radS mutant confirmed the expression of radR, while the reverse was not observed (data not shown). Further, we observed that the mere absence of RadS in the $\Delta \mathrm{radS}$ mutant was able to affect the expression of various genes in D. radiodurans exposed to gamma radiation (GEO accession numbers GSE17720, GSM442437, GSM442435). This might indicate that although RadS functions through RadR as its cognate response regulator, it also functions through other proteins, which possibly contribute to the expression of genes responsible for radiation resistance. Although further studies are required to test these hypotheses, the dose-dependent effects of TCSs and the functional redundancy of such proteins have been reported in bacteria. These results therefore suggested that RadS and RadR are expressed in a bicistronic operon and have roles in the radiation resistance of $D$. radiodurans.

\section{Both the $\Delta$ radS mutant and the $\Delta r a d R$ mutant show defective DSB reassembly}

To understand the molecular mechanisms underlying the roles of these proteins in the radiation resistance of $D$. radiodurans, the DSB repair of $\Delta \mathrm{rad} S$ and $\Delta \mathrm{rad} R$ mutants was monitored by PFGE. Interestingly, although the two mutants showed a nearly identical response to gamma radiation, for unknown reasons the DSB repair kinetics were slightly different. The $\Delta \mathrm{rad} S$ mutant showed a delayed recovery of the NotI pattern of its genome as compared with the wild-type, while the $\Delta r a d R$ mutant showed the faster appearance of several NotI fragments, although the pattern (Mattimore \& Battista, 1996) was different from that of the wild-type (Fig. 5): at least one large band and a few smaller-size NotI fragments were missing. This may indicate a genomic rearrangement that has made the radR deletion mutant sensitive to gamma radiation. PFGE carried out with undigested genomic DNA supported this (a) Wild-type
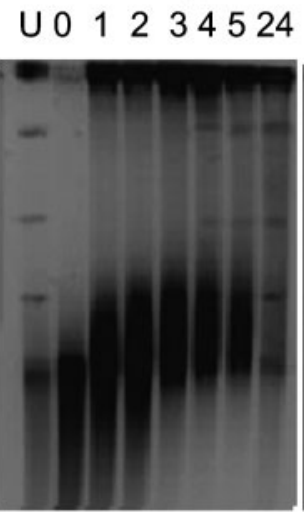

(b) Wild-type

U $01234524 \mathrm{M}$

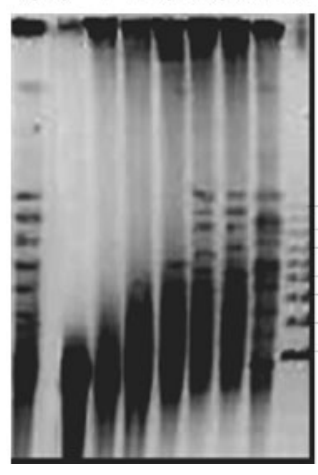

$\Delta r a d S$

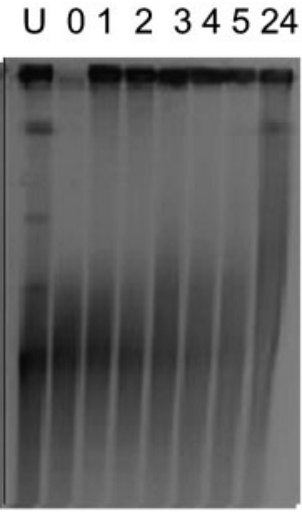

$\Delta$ rads

U 01234524 MU 01234524

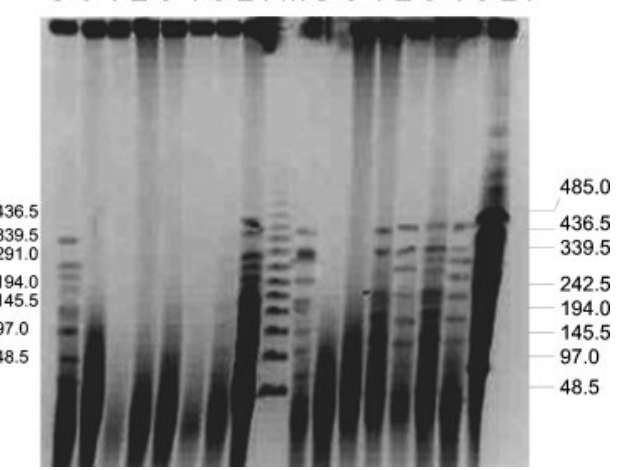

$\Delta$ radR

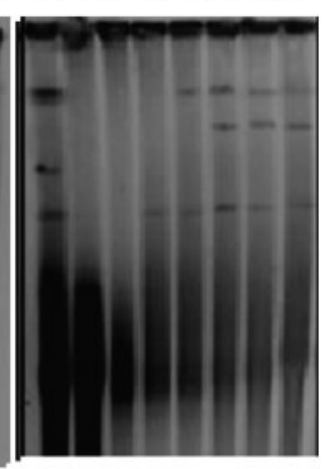

$\Delta$ radR
Fig. 5. DNA strand break repair kinetics during PIR recovery. The wild-type and deletion mutants of the radS ( $\Delta \mathrm{rad} S$ ) and radR $(\Delta \mathrm{rad} R)$ genes were treated with $6.5 \mathrm{kGy}$ of gamma radiation, and aliquots were drawn at different times $(0,1,2,3,4,5$ and 24 h) of PIR recovery. Both undigested (a) and Notldigested (b) genomic DNA was independently analysed by PFGE and compared with unirradiated controls $(U)$ and PFGE size markers (M). 
speculation. The patterns of DNA bands, presumably representing the banding of intact genomes in unirradiated cells of the wild-type, $\Delta \mathrm{rad} S$ and $\Delta \mathrm{radR}$ mutants, were similar (Fig. 5). Upon gamma irradiation followed by PIR recovery, these cells showed distinct patterns. The $\Delta \mathrm{rad} S$ mutant showed no sign of recovery, while the wild-type recovered its typical pattern. The $\Delta \mathrm{radR}$ mutant showed a different pattern of recovered DNA signatures (Fig. 5a, compare lanes 4, 5 and 24 of $\Delta \mathrm{radR}$ with those of the wildtype). These results might lead one to postulate a role for RadR in maintaining genome integrity during the PIR recovery of this bacterium. Recently, the possibility of genomic rearrangement in recombination repair mutants recovering from radiation stress has been indicated (Repar et al., 2010; Hickman et al., 2010). Delayed DSB repair leading to gamma radiation sensitivity as observed in the $\Delta r a d S$ mutant agrees with previous findings in which the loss of gamma radiation resistance was due to a defect in the DSB repair of D. radiodurans (Misra et al., 2006; Slade et al., 2009; Khairnar et al., 2008). The differential effects of radS and radR deletions on DSB repair, despite the fact that both mutants show a near-identical response to gamma radiation, is interesting and intriguing. Both these mutants showed a growth pattern almost identical to that of the wild-type under standard culture conditions (data not shown), suggesting that these deletions have an insignificant effect on the normal growth of this bacterium but do have a role in response to DNA damage.

\section{Recombinant RadR is phosphorylated by recombinant RadS in solution}

The recombinant RadS and RadR proteins were purified to near homogeneity (Fig. 6). The kinase activity of RadS was checked in the presence and absence of purified RadR as a probable substrate. RadS showed autophosphorylation in the presence of $\left[\gamma_{-}{ }^{33} \mathrm{P}\right] \mathrm{ATP}$, but RadR phosphorylation was observed only in the presence of RadS (Fig. 6). When increasing amounts of RadR were incubated with $\left[\gamma^{3}{ }^{33} \mathrm{P}\right] \mathrm{ATP}$ in the absence of RadS, the protein did not show phosphorylation (Fig. 6b), while RadR incubated with RadS showed phosphorylation. A time-course assay supported the conclusion that RadS phosphorylates RadR in vitro (Fig. 6c). These results suggested the functional interaction of RadS with RadR, and that RadR is a substrate for RadS in vitro. The level of RadS phosphorylation reduced significantly when it was incubated with a higher molar ratio of RadR (data not shown), and also on longer incubation with twice the molar concentration of RadR (Fig. 6c). An increased phosphorylation of RadR by RadS and a reduction in the signal of RadS both at the higher molar ratio of RadR and on longer incubation might argue in favour of substrate-driven forward kinetics for the phosphotransfer reaction catalysed by RadS. Surprisingly, the recombinant RadS purified from E. coli showed low activity in vitro. However, the low activity of a mycobacterial membrane histidine kinase purified from $E$. coli has been shown (Shrivastava et al., 2007). These results suggest that RadS is a (a)

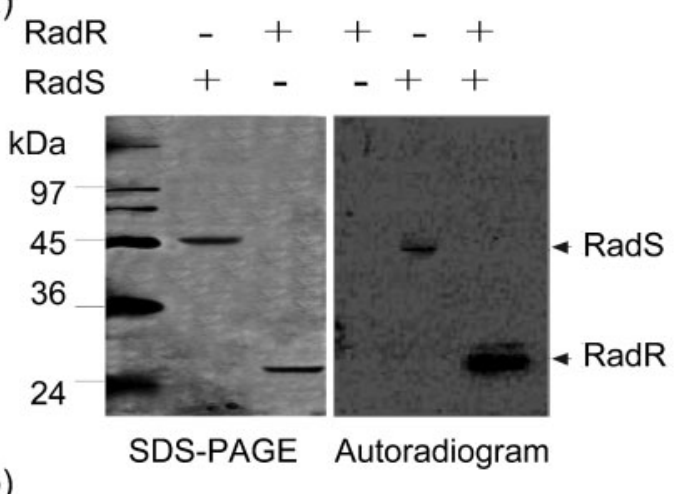

(b)

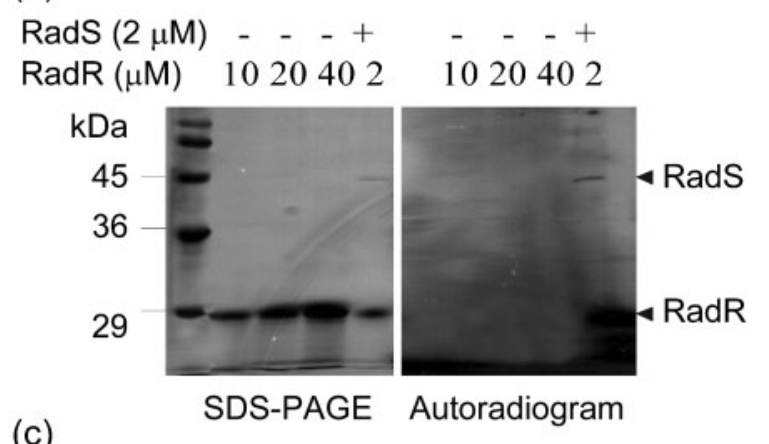

(c)

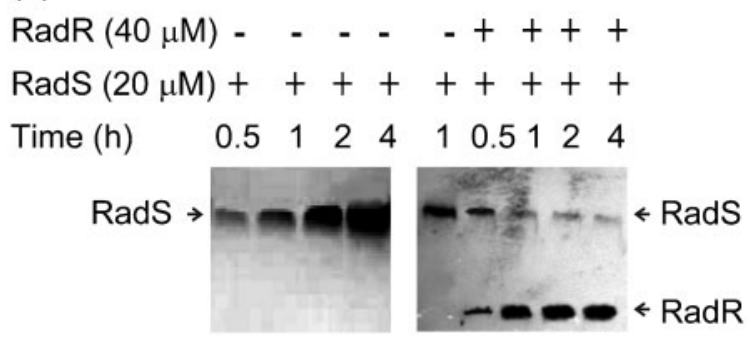

Fig. 6. Functional characterization of RadS and RadR proteins in vitro. Purified RadS was incubated with RadR in different combinations in the presence of $\left[\gamma^{33}\right.$ P PATP at $37^{\circ} \mathrm{C}$ for $6 \mathrm{~h}$ (a), and with increasing concentrations of RadR in the presence of [ $\gamma^{-32}$ P]ATP (b). To determine the time-dependent autokinase and transkinase activities of RadS, purified RadS was incubated alone and in the presence of RadR for different time intervals in the presence of $\left[\gamma^{33}\right.$ P]ATP (c). The products were analysed and proteins were visualized by Coomassie staining (SDS-PAGE), and phosphoproteins were visualized by autoradiography (Autoradiogram).

histidine kinase which can phosphorylate a cognate response regulator, RadR, expressed from the same operon.

Both membrane-bound and cytoplasmic signal transduction protein kinases and their cognate response regulators have been identified in bacteria (Parkinson, 1993; Mascher et al., 2006). Roles for histidine kinases and their cognate response regulators in sensing heavy metal stress by inducing the cascade of protein phosphorylation have been reported. $D$. radiodurans has been shown to accumulate a higher amount of $\mathrm{Mn}$ as compared with $\mathrm{Fe}$ and $\mathrm{Cu}$ (Ghosal et al., 2005), and the physiological significance of this in the 
bacterial response to oxidative and radiation stress has been reported (Shashidhar et al., 2010; Bagwell et al., 2008). RadS shows $37 \%$ identity to the histidine kinase CusS, involved in sensing copper toxicity in E. coli (Rensing \& Grass, 2003). Classical TCSs, consisting of a sensor protein kinase and a response regulator regulating the differential expression of a gene in response to nutritional and other abiotic stresses, with the exception of DNA damage, have been reported in bacteria (Parkinson, 1993; Inouye \& Dutta, 2003). On the other hand, the DNA damage response-mediated regulation of the cell cycle and DSB repair have been well documented in eukaryotes (Sancar et al., 2004; Lavin \& Kozlov, 2007).

Here, we report findings that suggest a role for a histidine kinase (RadS) and its cognate response regulator (RadR) in the high tolerance to DNA damage of $D$. radiodurans. Both autophosphorylation of RadS and its ability to phosphorylate RadR in vitro indicate the stress-responsive nature of the RadS/RadR system. Recently, the involvement of protein phosphorylation in DNA metabolism and radiation resistance has been shown in bacteria. The role of pyrroloquinolinequinone (PQQ), an inducer of protein kinase activity, in radiation resistance and DSB repair (Rajpurohit et al., 2008), the co-existence of DNA repair proteins with protein kinases and phosphoproteins in a multiprotein DNA-processing complex (Kota \& Misra, 2008), and the role of a response regulator, DR_2418, in the regulation of recA and pprA expression (Wang et al., 2008), have been reported in $D$. radiodurans. Also, a DNA damage-responsive membrane protein kinase with a role in radiation resistance and DSB repair has been characterized in D. radiodurans (Rajpurohit \& Misra, 2010), and gamma radiation-induced changes in the levels of cyclic AMP and ATP, and the activity of stressresponsive enzymes such as adenylyl cyclase, protein kinases and phosphodiesterases, have been shown in this bacterium (Kamble et al., 2010). The functional interaction of PQQ with a periplasmic protein kinase with a role in radiation tolerance (Khairnar et al., 2007), and the role of a TCS in the expression of DNA recombination and repair genes, have also been reported in E. coli (Oshima et al., 2002; Zhou et al., 2003). The phosphorylation of the single strand DNA-binding protein (SSB) of Bacillus subtilis and its effect on the DNA-binding activity of SSB have been demonstrated (Mijakovic et al., 2006). These findings support the involvement of protein phosphorylation and the roles of protein kinases in the bacterial response to DNA damage. The results presented here suggest that RadS/RadR form a TCS that contributes to the extraordinary tolerance of $D$. radiodurans to radiation resistance and DNA damage, and to our knowledge is the first report showing the role of a TCS in the radiation resistance of a prokaryote.

\section{ACKNOWLEDGEMENTS}

The authors thank Dr S. K. Apte, Bhabha Atomic Research Centre, and Professor Deepti D. Deobagkar, University of Pune, for their deep interest in the subject and technical comments while pursuing this work. Mass spectrometric analysis of polypeptides was carried out at the Mass Spectrometry facility at the Indian Institute of Science, Bangalore, India. S. S. D. thanks the Bhabha Atomic Research Centre, Department of Atomic Energy, India, for providing a research fellowship.

\section{REFERENCES}

Appleby, J. L., Parkinson, J. S. \& Bourret, R. B. (1996). Signal transduction via the multi-step phosphorelay: not necessarily a road less traveled. Cell 86, 845-848.

Aravind, L. \& Koonin, E. V. (1999). DNA polymerase $\beta$-like nucleotidyltransferase superfamily: identification of three new families, classification and evolutionary history. Nucleic Acids Res 27, 1609-1618.

Bagwell, C. E., Milliken, C. E., Ghoshroy, S. \& Blom, D. A. (2008). Intracellular copper accumulation enhances the growth of Kineococcus radiotolerans during chronic irradiation. Appl Environ Microbiol 74, 1376-1384.

Battista, J. R., Park, M. J. \& McLemore, A. E. (2001). Inactivation of two homologues of proteins presumed to be involved in the desiccation tolerance of plants sensitizes Deinococcus radiodurans $\mathrm{R} 1$ to desiccation. Cryobiology 43, 133-139.

Blasius, M., Sommer, S. \& Hübscher, U. (2008). Deinococcus radiodurans: what belongs to the survival kit? Crit Rev Biochem Mol Biol 43, 221-238.

Bonacossa de Almeida, C., Coste, G., Sommer, S. \& Bailone, A. (2002). Quantification of RecA protein in Deinococcus radiodurans reveals involvement of RecA, but not LexA, in its regulation. Mol Genet Genomics 268, 28-41.

Cox, M. M. \& Battista, J. R. (2005). Deinococcus radiodurans - the consummate survivor. Nat Rev Microbiol 3, 882-892.

Daly, M. J. \& Minton, K. W. (1996). An alternative pathway of recombination of chromosomal fragments precedes recA-dependent recombination in the radioresistant bacterium Deinococcus radiodurans. J Bacteriol 178, 4461-4471.

Daly, M. J., Ouyang, L., Fuchs, P. \& Minton, K. W. (1994). In vivo damage and recA-dependent repair of plasmid and chromosomal DNA in the radiation-resistant bacterium Deinococcus radiodurans. J Bacteriol 176, 3508-3517.

Ghosal, D. M., Omelchenko, M. V., Gaidamakova, E. K., Matrosova, V. Y., Vasilenko, A., Venkateswaran, A., Zhai, M., Kostandarithes, H. M., Brim, H. \& other authors (2005). How radiation kills cells: survival of Deinococcus radiodurans and Shewanella oneidensis under oxidative stress. FEMS Microbiol Rev 29, 361-375.

Hickman, A. B., James, J. A., Barabas, O., Pasternak, C., Ton-Hoang, B., Chandler, M., Sommer, S. \& Dyda, F. (2010). DNA recognition and the precleavage state during single-stranded DNA transposition in D. radiodurans. EMBO J 29, 3840-3852.

Inouye, M. \& Dutta, R. (2003). Histidine Kinases in Signal Transduction. San Diego, CA: Academic Press.

Joshi, B., Schmid, R., Altendorf, K. \& Apte, S. K. (2004). Protein recycling is a major component of post-irradiation recovery in Deinococcus radiodurans strain R1. Biochem Biophys Res Commun 320, 1112-1117.

Kamble, V. A., Rajpurohit, Y. S., Srivastava, A. K. \& Misra, H. S. (2010). Increased synthesis of signaling molecules coincides with reversible inhibition of nucleolytic activity during postirradiation recovery of Deinococcus radiodurans. FEMS Microbiol Lett 303, 18-25.

Khairnar, N. P., Kamble, V. A., Mangoli, S. H., Apte, S. K. \& Misra, H. S. (2007). Involvement of a periplasmic protein kinase in DNA strand break repair and homologous recombination in Escherichia coli. Mol Microbiol 65, 294-304. 
Khairnar, N. P., Kamble, V. A. \& Misra, H. S. (2008). RecBC enzyme overproduction affects $\mathrm{UV}$ and gamma radiation survival of Deinococcus radiodurans. DNA Repair (Amst) 7, 40-47.

Kim, D. \& Forst, S. (2001). Genomic analysis of the histidine kinase family in bacteria and archaea. Microbiology 147, 1197-1212.

Kota, S. \& Misra, H. S. (2006). PprA: a protein implicated in radioresistance of Deinococcus radiodurans stimulates catalase activity in Escherichia coli. Appl Microbiol Biotechnol 72, 790-796.

Kota, S. \& Misra, H. S. (2008). Identification of a DNA processing complex from Deinococcus radiodurans. Biochem Cell Biol 86, 448-458.

Lavin, M. F. \& Kozlov, S. (2007). ATM activation and DNA damage response. Cell Cycle 6, 931-942.

Leonard, C. J., Aravind, L. \& Koonin, E. V. (1998). Novel families of putative protein kinases in bacteria and archaea: evolution of the "eukaryotic" protein kinase superfamily. Genome Res 8, 1038-1047.

Liu, Y., Zhou, J., Omelchenko, M. V., Beliaev, A. S., Venkateswaran, A., Stair, J., Wu, L., Thompson, D. K., Xu, D. \& other authors (2003). Transcriptome dynamics of Deinococcus radiodurans recovering from ionizing radiation. Proc Natl Acad Sci U S A 100, 4191-4196.

Makarova, K. S., Aravind, L., Wolf, Y. I., Tatusov, R. L., Minton, K. W., Koonin, E. V. \& Daly, M. J. (2001). Genome of the extremely radiationresistant bacterium Deinococcus radiodurans viewed from the perspective of comparative genomics. Microbiol Mol Biol Rev 65, 44-79.

Markillie, L. M., Varnum, S. M., Hradecky, P. \& Wong, K. K. (1999). Targeted mutagenesis by duplication insertion in the radioresistant bacterium Deinococcus radiodurans. radiation sensitivities of catalase ( $k a t A$ ) and superoxide dismutase (sodA) mutants. J Bacteriol 181, 666-669.

Mascher, T., Helmann, J. D. \& Unden, G. (2006). Stimulus perception in bacterial signal-transducing histidine kinases. Microbiol Mol Biol Rev 70, 910-938.

Mattimore, V. \& Battista, J. R. (1996). Radioresistance of Deinococcus radiodurans: functions necessary to survive ionizing radiation are also necessary to survive prolonged desiccation. J Bacteriol 178, 633-637.

Meima, R., Rothfuss, H. M., Gewin, L. \& Lidstrom, M. E. (2001). Promoter cloning in the radioresistant bacterium Deinococcus radiodurans. J Bacteriol 183, 3169-3175.

Mijakovic, I., Petranovic, D., Macek, B., Cepo, T., Mann, M., Davies, J., Jensen, P. R. \& Vujaklija, D. (2006). Bacterial single-stranded DNA-binding proteins are phosphorylated on tyrosine. Nucleic Acids Res 34, 1588-1596.

Misra, H. S., Pandey, P. K., Modak, M. J., Vinayak, R. \& Pandey, V. N. (1998). Polyamide nucleic acid-DNA chimera lacking the phosphate backbone are novel primers for polymerase reaction catalyzed by DNA polymerases. Biochemistry 37, 1917-1925.

Misra, H. S., Khairnar, N. P., Kota, S., Shrivastava, S., Joshi, V. P. \& Apte, S. K. (2006). An exonuclease I-sensitive DNA repair pathway in Deinococcus radiodurans: a major determinant of radiation resistance. Mol Microbiol 59, 1308-1316.

Narumi, I., Satoh, K., Kikuchi, M., Funayama, T., Yanagisawa, T., Kobayashi, Y., Watanabe, H. \& Yamamoto, K. (2001). The LexA protein from Deinococcus radiodurans is not involved in RecA induction following gamma irradiation. J Bacteriol 183, 6951-6956.

Oshima, T., Aiba, H., Masuda, Y., Kanaya, S., Sugiura, M., Wanner, B. L., Mori, H. \& Mizuno, T. (2002). Transcriptome analysis of all twocomponent regulatory system mutants of Escherichia coli K-12. Mol Microbiol 46, 281-291.

Parkinson, J. S. (1993). Signal transduction schemes of bacteria. Cell 73, 857-871.

Rajpurohit, Y. S. \& Misra, H. S. (2010). Characterization of a DNA damage-inducible membrane protein kinase from Deinococcus radiodurans and its role in bacterial radioresistance and DNA strand break repair. Mol Microbiol 77, 1470-1482.

Rajpurohit, Y. S., Gopalakrishnan, R. \& Misra, H. S. (2008). Involvement of a protein kinase activity inducer in DNA double strand break repair and radioresistance of Deinococcus radiodurans. J Bacteriol 190, 3948-3954.

Rensing, C. \& Grass, G. (2003). Escherichia coli mechanisms of copper homeostasis in a changing environment. FEMS Microbiol Rev 27, 197-213.

Repar, J., Cvjetan, S., Slade, D., Radman, M., Zahradka, D. \& Zahradka, K. (2010). RecA protein assures fidelity of DNA repair and genome stability in Deinococcus radiodurans. DNA Repair (Amst) 9, 1151-1161.

Sambrook, J. \& Russell, D. W. (2001). Molecular Cloning: a Laboratory Manual, 3rd edn. Cold Spring Harbor, NY: Cold Spring Harbor Laboratory.

Sancar, A., Lindsey-Boltz, L. A., Unsal-Kaçmaz, K. \& Linn, S. (2004). Molecular mechanisms of mammalian DNA repair and the DNA damage checkpoints. Annu Rev Biochem 73, 39-85.

Shashidhar, R., Kumar, S. A., Misra, H. S. \& Bandekar, J. R. (2010). Evaluation of the role of enzymatic and nonenzymatic antioxidant systems in the radiation resistance of Deinococcus. Can J Microbiol 56, 195-201.

Shimoni, Y., Altuvia, S., Margalit, H. \& Biham, O. (2009). Stochastic analysis of the SOS response in Escherichia coli. PLoS ONE 4, e5363.

Shrivastava, R., Ghosh, A. K. \& Das, A. K. (2007). Probing the nucleotide binding and phosphorylation by the histidine kinase of a novel three-protein two-component system from Mycobacterium tuberculosis. FEBS Lett 581, 1903-1909.

Slade, D. \& Radman, M. (2011). Oxidative stress resistance in Deinococcus radiodurans. Microbiol Mol Biol Rev 75, 133-191.

Slade, D., Lindner, A. B., Paul, G. \& Radman, M. (2009). Recombination and replication in DNA repair of heavily irradiated Deinococcus radiodurans. Cell 136, 1044-1055.

Tanaka, M., Earl, A. M., Howell, H. A., Park, M. J., Eisen, J. A., Peterson, S. N. \& Battista, J. R. (2004). Analysis of Deinococcus radiodurans's transcriptional response to ionizing radiation and desiccation reveals novel proteins that contribute to extreme radioresistance. Genetics 168, 21-33.

Walker, G. C. (1996). The SOS response of Escherichia coli. In Escherichia coli and Salmonella: Cellular and Molecular Biology, 2nd edn, pp. 1400-1416. Edited by F. C. Neidhardt \& other authors. Washington, DC: American Society for Microbiology.

Wang, L., Xu, G., Chen, H., Zhao, Y., Xu, N., Tian, B. \& Hua, Y. (2008). DrRRA: a novel response regulator essential for the extreme radioresistance of Deinococcus radiodurans. Mol Microbiol 67, 1211-1222.

White, O., Eisen, J. A., Heidelberg, J. F., Hickey, E. K., Peterson, J. D., Dodson, R. J., Haft, D. H., Gwinn, M. L., Nelson, W. C. \& other authors (1999). Genome sequence of the radioresistant bacterium Deinococcus radiodurans $\mathrm{R} 1$. Science 286, 1571-1577.

Zahradka, K., Slade, D., Bailone, A., Sommer, S., Averbeck, D., Petranovic, M., Lindner, A. B. \& Radman, M. (2006). Reassembly of shattered chromosomes in Deinococcus radiodurans. Nature 443, 569-573.

Zhou, B. B. \& Elledge, S. J. (2000). The DNA damage response: putting checkpoints in perspective. Nature 408, 433-439.

Zhou, L., Lei, X. H., Bochner, B. R. \& Wanner, B. L. (2003). Phenotype microarray analysis of Escherichia coli K-12 mutants with deletions of all two-component systems. J Bacteriol 185, 4956-4972.

Edited by: J. Green 